\title{
El tabaquismo se asocia a mayor riesgo de desarrollar demencia vascular y enfermedad de Alzheimer
}

Smoking is associated with an increased risk of developing vascular dementia and Alzheimer's disease

Rusanen M, y col. Arch Intern Med. 2011,171(4):333-339

\section{Objetivos}

Evaluar la asociación entre la cantidad de cigarrillos que se fuman en la mitad de la vida y el riesgo de desarrollar demencia dos o tres décadas después, Enfermedad de Alzheimer (EA) y Demencia vascular (DV).

\section{Diseño, lugar y pacientes}

Cohorte prospectiva multiétnica llevada a cabo en clínicas de San Francisco y Oakland, California. Ingresaron voluntariamente entre 1978 y 1985 miembros del Programa de Cuidado Médico Kaiser Permanente de California del Norte, cuando tenían entre 50 y 60 años de edad $(n=33.108)$.

Fueron seguidos hasta 1994, año en el cual sobrevivían 21.123 personas.

Tabla 1: tasa de incidencia de demencia, enfermedad de Alzheimer y demencia vascular de acuerdo al estatus de fumador en la mitad de la vida, ajustada por potenciales confundidores ${ }^{a}$

\begin{tabular}{|c|c|c|c|c|c|c|c|}
\hline \multirow[b]{2}{*}{ Estatus del fumador } & \multicolumn{3}{|c|}{ Sindrome demencial } & \multicolumn{2}{|c|}{ Enfermedad de Alzheimer } & \multicolumn{2}{|c|}{ Demencia vascular } \\
\hline & n & $\begin{array}{c}\text { TIE por } 10000 \\
\text { personas/año (IC95\%) }\end{array}$ & HR (IC 95\%) & $\mathbf{n}$ & HR1 (IC 95\%) & n & HR1 (IC 95\%) \\
\hline Nunca & 2724 & $\begin{array}{c}409,03 \\
(392,02 \text { a } 426,03)\end{array}$ & 1 & 590 & 1 & 210 & 1 \\
\hline Ex-tabaquista & 1628 & $\begin{array}{c}403,08 \\
(381,05 \text { a } 425,11)\end{array}$ & $\begin{array}{c}1 \\
(0,94 \text { a } 1,07)\end{array}$ & 351 & $\begin{array}{c}1 \\
(0,89 \text { a } 1,13)\end{array}$ & 124 & $\begin{array}{c}0,99 \\
(0,88 \text { a } 1,22)\end{array}$ \\
\hline $\begin{array}{c}\text { Menor a 0,5 } \\
\text { (paq/dia) }\end{array}$ & 234 & $\begin{array}{c}398,19 \\
(337,64 \text { a 458,75) }\end{array}$ & $\begin{array}{c}1,04 \\
(0,91 \text { a } 1,20)\end{array}$ & 39 & $\begin{array}{c}0,80 \\
(0,61 \text { a } 1,06)\end{array}$ & 17 & $\begin{array}{c}1,05 \\
(0,69 \text { a } 1,61)\end{array}$ \\
\hline $\begin{array}{c}0,5 \text { a } 1 \\
\text { (paq/dia) }\end{array}$ & 435 & $\begin{array}{c}483,59 \\
(425,64 \text { a } 541,54)\end{array}$ & $\begin{array}{c}1,37 \\
(1,23 \text { a } 1,52)\end{array}$ & 89 & $\begin{array}{c}1,11 \\
(0,90 \text { a } 1,36)\end{array}$ & 31 & $\begin{array}{c}1,20 \\
(0,84 \text { a } 1,7)\end{array}$ \\
\hline $\begin{array}{c}1 \text { a } 2 \\
\text { (paq/dia) }\end{array}$ & 283 & $\begin{array}{c}489,14 \\
(410,44 \text { a } 567,85)\end{array}$ & $\begin{array}{c}1,44 \\
(1,26 \text { a } 1,64)\end{array}$ & 50 & $\begin{array}{c}1,18 \\
(0,92 \text { a } 1,52)\end{array}$ & 29 & $\begin{array}{c}1,42 \\
(0,95 \text { a } 2,13)\end{array}$ \\
\hline $\begin{array}{c}\text { Mayor a } 2 \\
\text { (paq/dia) }\end{array}$ & 63 & $\begin{array}{c}786,42 \\
(481,23 \text { a } 1091,6)\end{array}$ & $\begin{array}{c}2,14 \\
(1,65 \text { a } 2,78)\end{array}$ & 17 & $\begin{array}{c}2,57 \\
(1,63 \text { a } 4,03)\end{array}$ & 5 & $\begin{array}{c}2,72 \\
(1,20 \mathrm{a} 6,18)\end{array}$ \\
\hline
\end{tabular}

TIE: tasa de incidencia ajustada por la edad. EA: Enfermedad de Alzheimer. DV: demencia vascular. HR: hazard ratio. a. Ajustada por edad, sexo, educación, raza, estado civil, hipertensión arterial, hiperlipidemia, IMC, diabetes, enfermedad coronaria, ACV y consumo de alcohol.

\section{Conclusiones}

El tabaquismo en la edad media se asoció con mayor riesgo de desarrollar demencia, enfermedad de Alzheimer y demencia vascular luego de dos décadas.
Evaluación de factores pronósticos y medición de resultados principales

Fueron evaluados los hábitos de vida (incluido tabaquismo), el índice de masa corporal (IMC), la tensión arterial (TA) y los lípidos, así como parámetros del metabolismo de la glucemia, antecedes personales y familiares de hipertensión arterial, diabetes y accidente cerebrovascular (ACV). Todos los diagnósticos fueron confirmados desde 1994 (media de edad: 71,6 $\pm 5,8$ años) hasta 2008. Los diagnósticos sindromáticos de demencia fueron realizados por médicos internistas, y los de EA y DV por neurólogos o neuropsicólogos.

\section{Resultados Principales}

5367 personas $(25,4 \%)$ tuvieron diagnóstico de demencia (1136 EA y 416 DV) durante un seguimiento medio de 23 años. Los resultados principales se describen en la tabla 1.

\section{Comentario}

En Argentina, el consumo de tabaco es una de las principales causa de muerte por enfermedad cardiovascular, pulmonar y cáncer ${ }^{1}$, y según la Encuesta Nacional de Factores de Riesgo de 2009 , la prevalencia del consumo de tabaco es del $27,1 \%$, cifras que muestran una escasa disminución con respecto de $2005(29,7 \%)^{1}$. El tabaquismo es un conocido factor de riesgo para ACV, lo que implica mayor predisposición a demencia multi-infarto, mientras que su asociación con EA es controvertida. Los resultados del estudio de Rusanen que hemos resumido muestran asociación entre el tabaquismo y ambas formas de demencia, pudiéndose observar cierto gradiente dosis-
Palabras claves: cohorte prospectiva, fumadores, demencia, enfermedad de Alzheimer, demencia vascular. Key words: prospective cohorte, smokers, dementia, Alzheimer disease, vascular dementia. Fuente de financiamiento: Escuela Nacional Superior de Investigación Clínica, Hospital Universitario Kuopio, Fundación Juho Vainio, Fundación Maire Taponen, Kaiser Permanente, Instituto Nacional de Salud y Academia de Finlandia.

Mercedes Mutchinick [ Servicio de Medicina Familiar y Comunitaria del Hospital Italiano de Buenos Aires. mercedes.mutchinick@ hospitalitaliano.org.ar ]

respuesta que refuerza la hipótesis de causalidad ${ }^{3}$, especialmente cuando el consumo supera diez cigarrillos diarios.

\section{Conclusiones de la comentadora}

El tabaquismo activo durante la mitad de la vida se asocia a un aumento del riesgo de demencia (EA y DV) en ambos sexos y en diferentes grupos raciales. Tenemos una razón más para advocar a las autoridades políticas medidas tendientes al control de esta pandemia, para desaconsejarlo y para ofrecer ayuda a nuestros pacientes fumadores.

Mutchinik M. El tabaquismo se asocia a mayor riesgo de desarrollar demencia vascular y enfermedad de Alzheimer. Evid Act Pract Ambul Jul-Set 2011;14(3):96. Comentado de: Rusanen M y col. Heavy Smoking in Midlife and Long-term Risk of Alzheimer Disease and Vascular Dementia. Arch Intern Med. Published online October 25, 2010. doi:10.1001/archinternmed.2010.393. PMID: 20975015.

\section{Referencias}

1. Benowitz N. Nicotine Adiction. NEJM. Junio 17, 2010; 362(24): 2295-2303.

2. Ferrante y col. Encuesta nacional de factores de riesgo 2009: evolución de la epidemia de enfermedades crónicas no transmisibles en argentina. Estudio de corte transversal. Rev Argent Salud Pública, 2011; 2(6):34-41

3. Austin Bradford Hill, "The Environment and Disease: Association or Causation?" Proceedings of the Royal Society of Medicine, 58 (1965), 295-300. Disponible en URL: http://www.edwardtufte.com/turte/hill 